\title{
Hybrid Polymer/MOF membranes for Organic Solvent Nanofiltration (OSN): Chemical Modification and the Quest for Perfection
}

James Campbell ${ }^{a}$, Joao Da Silva Burgal ${ }^{a}$, Gyorgy Szekely ${ }^{a}$, R.P. Davies ${ }^{b}$, D. Christopher Braddock ${ }^{b}$, Andrew Livingston ${ }^{a, *}$

${ }^{\text {a }}$ Department of Chemical Engineering, Imperial College, London SW7 2AZ, UK

${ }^{b}$ Department of Chemistry, Imperial College, London SW7 2AZ, UK

*Corresponding Author:

Andrew Livingston, Tel: +44 (0)20 7594 5582. E-mail: a.livingston@imperial.ac.uk.

\begin{abstract}
One of the main challenges in the field of organic solvent nanofiltration (OSN) is to improve the selectivity of membranes, allowing the separation of closely related solutes. This objective might be achieved by constructing membranes with uniform porous structures. Hybrid Polymer/Metal Organic Framework (MOF) membranes were prepared by in-situ growth (ISG) of HKUST-1 within the pores of polyimide membranes. To improve the performances of ISG membranes, chemical modification was performed. Aryl carboxylic acid moieties were introduced to polyimide P84 ultrafiltration membranes allowing coordination of the HKUST-1 directly on to the polymer. Chemically modified ISG membranes outperformed non-modified ISG membranes in both solute retentions and permeance. Retentions of polystyrene solute in acetone were used to calculate theoretical pore size distributions for each of the membranes tested. It was found that the chemically modified ISG membrane had he narrowest calculated pore size distribution.
\end{abstract}

Keywords: Hybrid Membranes, Organic Solvent Nanofiltration, Metal Organic Frameworks, Chemical Modification, Modelling

\section{Abbreviations}

CMA Chemical Modification Agent

HKUST Hong Kong University of Science and Technology

HDA Hexane-1,6-diamine

ISA Integrally skinned asymmetric (membrane)

ISG In-Situ Growth

MMM Mixed Matrix Membrane

MOF Metal Organic Framework

OSN Organic Solvent Nanofiltration

SEM Scanning Electron Microscopy

TFC Thin Film Composite 


\section{Introduction}

Organic solvent nanofiltration (OSN) is an emerging pressure driven separation methodology that uses semi-permeable membranes to selectively separate on a molecular basis[1, 2]. Polymeric integrally skinned asymmetric (ISA) membranes fabricated via immersion precipitation phase inversion remain the most commonly used membranes in OSN processes[2]. Polymeric ISA membranes have a number of advantages, including flexibility and ease of production. The properties of polymer membranes can be altered by modifying the phase inversion process[3], however the structure of the selective layer of ISA membranes cannot yet be designed at a molecular level. ISA membranes will always exhibit a pore size distribution, rather than a single regular pore size[4]. To introduce more molecular order to the structure of polymeric membranes, hybrid membranes containing metal organic frameworks (MOFs) have been developed.

Recent developments in the fabrication of hybrid polymer/MOF membranes have led to the development of these kinds of membranes for gas separations and OSN processes. Mixed matrix membranes (MMM) have been shown to improve the permeance and selectivity of polymeric membranes above the Robeson upper bound for gas separations[5-12]. MMMs have also been used for OSN processes, using various MOF particles embedded in the selective layer of thin film composite (TFC) membranes[13], and HKUST-1 particles dispersed in integrally skinned asymmetric (ISA) polymer membranes[14]. While MMMs have been shown to improve the retention properties of polymer membranes further improvements were made using in-situ growth (ISG) membranes.

In-situ growth (ISG) is a hybrid membrane fabrication methodology which has been used to produce hybrid polyimide/HKUST-1 membranes for OSN[14]. ISG membranes have been shown to improve solute retentions and flux decline properties above that of pure polymer membranes and mixed matrix membranes (MMMs) for OSN. ISG membranes have also been used for gas separations[1517]. However there is scope for further improvement of the performances of ISG hybrid membranes through modification of the polymer matrix. Chemical modification techniques have been successfully applied to MMMs containing inorganic particles to improve the interaction between the polymer and inorganic phases of the membranes for gas separations[18, 19] and OSN[13, 20].

Functionalization of polymers by small-molecule organic chemistry reactions is a vibrant field of research and has attracted great attention lately due to an increasing number of novel potential applications[21]. Post-polymerization chemical modification can be used to incorporate functionalities into existing polymer chains and networks. This approach circumvents a number of problems associated with direct polymer synthesis and enables the creation of polymeric systems which may be difficult or impossible to produce otherwise. Examples include post-polymerization modifications to create aliphatic poly(carbonate)s for biomedical applications[22], imprinted materials for advanced separations[23] or functional polymers featuring labile groups such as alkynes or thiols[24]. In order to achieve improved solute retentions using ISG membranes, chemical modification agents were used with a view to reducing the voids/defects between polymer and the MOF crystals. In this paper chemical modification has been applied to encourage the growth of MOF (HKUST-1) directly on the walls of the pores. This is achieved through the incorporation of benzene tri-carboxylate functional groups into the polyimide polymer chain. 

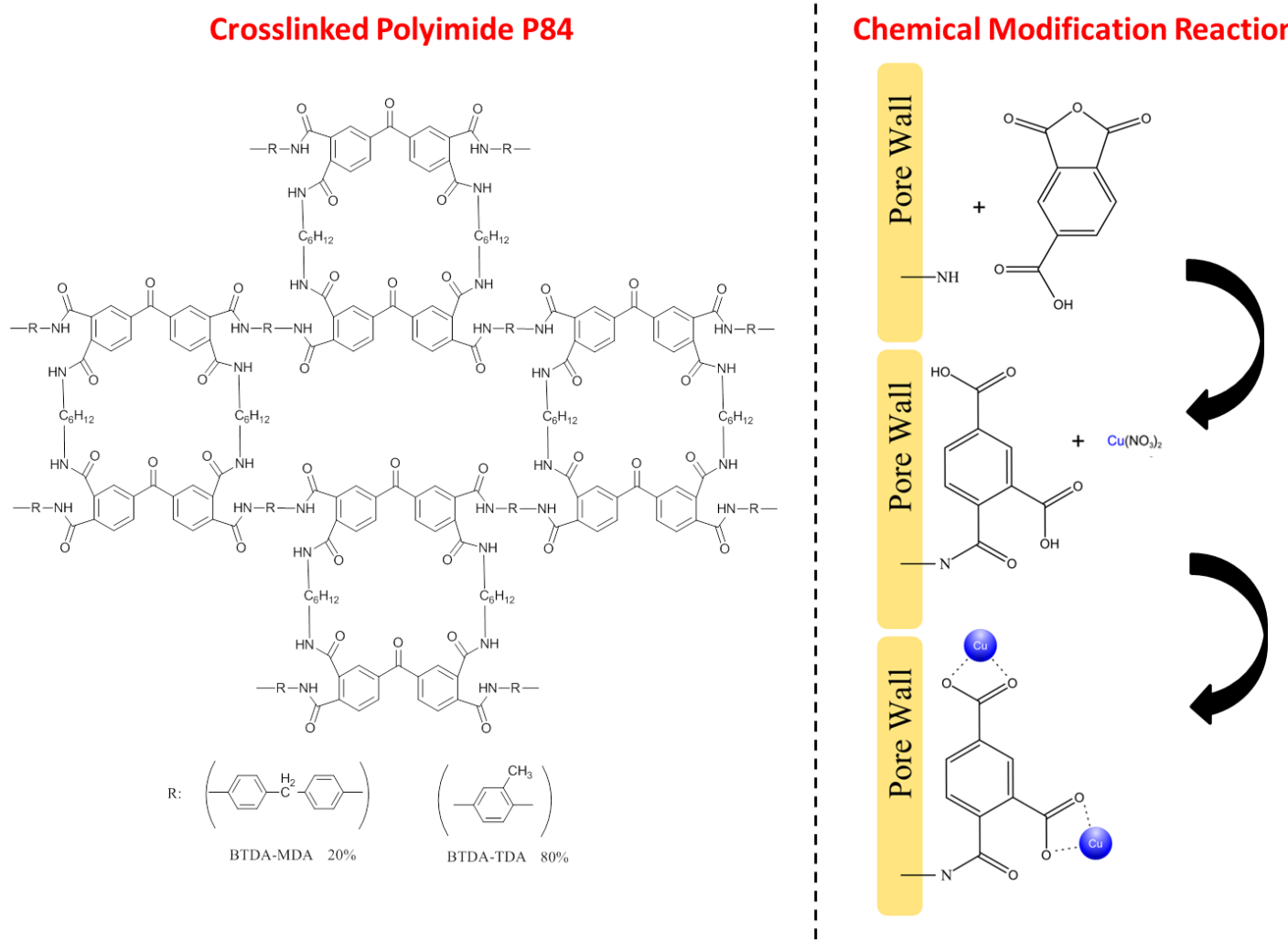

Figure 1: (Left) Crosslinked Polyimide P84. The opening of the imide ring during crosslinking with hexane-1,6-diamine (HDA)[25]leaves the polymer structure with four secondary amide units per monomer unit available to react with the CMA 1,2,4-benzenetricarboxylic anhydride. (Right) Schematic representation showing the introduction of a BTC moiety on a membrane surface, which in turn provides a chemical anchor for HKUST-1 growth.

The ISG membrane fabrication methodology was developed to modify the pores of ISA polymer membranes, intending to create membranes with more regular, predictable porous structures. Due to the phase inversion process, which is the most common methodology used to produce polymer membranes for OSN[2], ISA polymer membranes always exhibit a distribution of pore sizes[4, 26-28]. Introduction of HKUST-1 to hybrid polymer/MOF membranes is meant to augment the structure of the separation layer of the membrane and specifically to narrow its pore size distribution. Narrower pore size distributions could lead to sharper molecular weight cut-off (MWCO) curves, which would allow for better separations between molecules close in molecular weight.

It is difficult to directly measure, on a nanoscale, the pores in polymer materials experimentally. Recently methodologies have been developed, including Positron Anilhilation Lifetime Spectroscopy (PALS)[29, 30] and nanoprobe imaging[31]. However these methods are relatively unproven, and require expensive equipment and complicated techniques. Alternatively derivative methodologies can be employed, using filtration data to estimate pore size distributions. This paper uses experimental data to model the pore size distributions of the membranes in order to measure the effect MOF addition has on the pore size distribution of hybrid polymer/MOF membranes. Transport phenomena through membranes have been described using four kinds of models: irreversible thermodynamics, solution-diffusion, pore-flow and solution-diffusion with imperfections (SDI). The solution-diffusion and pore flow model are both widely used for describing 
transport through nanofiltration membranes [32]. In this paper the pore-flow model was chosen to study the effect chemical modification and the presence of MOFs on the pore size distribution of hybrid polymer/MOF membranes. Using the rejection of polystyrene solutes log-normal pore size probability functions were calculated for each of the membranes tested in this paper.

This paper uses the same pore flow model to predict the rejection and permeance of defect free HKUST-1 films. These calculations will evaluate how close ISG membranes have come to the performance of ideal MOF films.

\section{Experimental}

\subsection{Materials}

Polypropylene non-woven backing was supplied by Viledon, Germany. Polyimide (PI) polymer (P84) was purchased from HP Polymer $\mathrm{GmbH}$, Austria. Solvents used for membrane preparation and membrane testing including isopropanol, acetone, DMF, 1,4-dioxane, ethanol (99.7\%) and polyethylene glycol (MW 400) (PEG-400) were obtained from VWR international. Hexane-1,6diamine for crosslinking was purchased from Sigma Aldrich. Copper nitrate trihydrate and 1,3,5benzenetricarboxylic acid used to fabricate ISG hybrid polymer/MOF membranes were also purchased from VWR international. 1,2,4-Benzenetricarboxylic anhydride chemical modification agent (CMA) was purchased from Sigma-Aldrich. Polystyrene markers for solute rejection evaluation were purchased from Agilent Technologies, UK. Novatex 2471 Polypropylene non-woven supports were supplied by Freudenberg Filtration Technologies. All the chemicals were used as received without any further purification.

\subsection{Membrane Preparation}

\subsubsection{Preparation of Ultrafiltration Membranes}

Polyimide P84 ultrafiltration (UF) membranes were produced via phase inversion. UF membranes were fabricated via the methodology used to produce dense UF membranes previously described by the authors[14]. Dope solutions were formed by dissolving $24 \mathrm{wt} \%$ of polyimide polymer in DMF. The P84 was dissolved using a magnetic stirrer at room temperature, in a sealed container to ensure no moisture was absorbed into the solution. The dope solutions were cast onto polypropylene nonwoven supports using a casting knife set to a thickness of $250 \mu \mathrm{m}$, in an environment with a temperature of $15{ }^{\circ} \mathrm{C}$ and a humidity of $50-60 \%$. The membranes were then precipitated from solution via immersion in water. The membranes were subsequently placed in isopropyl alcohol (IPA) to remove water from the polymer matrix. The membranes were then submerged in $30 \mathrm{~g} \cdot \mathrm{L}^{-1}$ solutions of 1,6-hexamethylenediamine (HDA) in IPA for 20 hours in order to crosslink the polymer chains, improving the chemical resistance of the membranes. After crosslinking, the membranes were washed with IPA to remove excess crosslinking agent. Before testing, the membranes were conditioned with a PEG400:IPA (60:40 v/v) solution for 12 hours

\subsubsection{Membrane Chemical Modification}

Chemical modification was performed on UF membranes using the chemical modification agent (CMA) 1,2,4-benzenetricarboxylic anhydride in order to introduce a tricarboxylate functional group into the membrane matrix. Crosslinked polyimide P84 membranes contain amides, which can 
readily react with anhydrides. When the anhydride reacts with the amide the anhydride ring opens up and attaches to the polymer matrix, while also forming a carboxylate functional group available to react with copper ions to begin forming the building blocks of HKUST-1 within the membrane.

Prior to chemical modification the crosslinked UF membranes were washed with DMF to remove IPA from the pores. The membranes were placed in solutions of 1,2,4-benzenetricarboxylic anhydride in DMF for 20 hours at $25^{\circ} \mathrm{C}$. The amount of CMA added to the solutions was based on the assumption that each polymer unit contains four sites available to react with the 1,2,4-benzenetricarboxylic anhydride molecule (see Figure 1). For each membrane one equivalent of CMA was added using 10 g. $\mathrm{L}^{-1}$ solutions of 1,2,4-benzenetricarboxylic anhydride in DMF, with the volume of solution adjusted according to the mass of membrane. After the reaction each membrane was repeatedly washed with $\mathrm{DMF}$ to remove any unreacted CMA from the polymer matrix.

\subsubsection{Preparation of Hybrid Polymer/Metal Organic Membranes}

ISG hybrid polymer/MOF membranes were prepared by immersing the polymer UF membranes, both modified and unmodified, into a fresh mixture of copper nitrate ( $0.86 \mathrm{M} \mathrm{EtOH}$ solution) and 1,3,5-benzenetricarboxylic acid (0.40 M DMF solution) solution. The membranes were left in solution for 48 hours under occasional stirring and were then extensively washed with DMF to remove any unreacted reagents. Afterwards the membranes were conditioned by immersion in a PEG400:IPA $(60: 40 \mathrm{v} / \mathrm{v})$ solution for 12 hours.

Table 1: Membranes tested and the modifications attributed to each

\begin{tabular}{|c|c|c|c|}
\hline & \multicolumn{2}{|c|}{ HKUST-1 In-Situ Growth } \\
\hline & & No & Yes \\
\hline \multirow{2}{*}{ 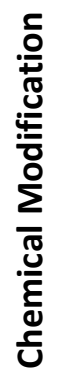 } & No & M1 & M2 \\
\hline & Yes & M3 & M4 \\
\hline
\end{tabular}

Four different types of membrane were fabricated so that the effects of chemical modification and ISG could be determined separately and in combination. Using a UF membrane, chemical modification was carried out on membranes M3 \& M4, while in-situ growth of HKUST-1 was carried out on membranes M2 \& M4. The details of all the membranes tested can be found in Table 1.

\subsubsection{Preparation of Polyimide (P84) Nanofiltration Membranes (M5)}

Polyimide P84 nanofiltration membranes were made using a similar procedure as that used to produce UF membranes, however for nanofiltration membranes the polymer was dissolved in a mixture of solvent/co-solvent as described by by See-Toh et al [4, 25]. For the polyimide nanofiltration membranes used in this study dope solutions were prepared by dissolving $24 \mathrm{wt} \%$ of polymer in DMF/1,4-dioxane solvent/co-solvent. The ratio of DMF:1,4-dioxane used was 1:1. The 
dope solution was stirred in a sealed container at room temperature until all the polymer had dissolved. Dope solutions were cast onto polypropylene non-woven sheets using a casting knife set to a thickness of $250 \mu \mathrm{m}$, in an environment with a temperature of $15^{\circ} \mathrm{C}$ and a humidity of $50-60 \%$. The membranes were then precipitated from solution via immersion in water. The membranes were subsequently placed in isopropyl alcohol (IPA) to remove water from the polymer matrix. The membranes were crosslinked using $30 \mathrm{~g} . \mathrm{L}^{-1}$ solutions of 1,6-hexamethylenediamine (HDA) in IPA for 20 hours. After crosslinking, the membranes were washed with IPA to remove excess crosslinking agent. Before testing, the membranes were conditioned with a PEG400:IPA (60:40 v/v) solution for 12 hours

\subsection{Membrane characterization}

Fourier Transform-Infrared (FT-IR) spectra were recorded on a Perkin-Elmer Spectrometer 100, with samples mounted on a zinc-selenium/diamond plate. The spectra were collected in the attenuated total reflection (ATR) mode, directly from the membrane surface. The spectra were recorded at a resolution of $4 \mathrm{~cm}^{-1}$ as an average of 16 scans. The membranes were washed in isopropanol to remove any contamination and dried before the analysis. The surfaces and cross-sections of the membrane were characterized by Scanning Electron Microscopy (SEM). The samples were sputtered with chromium under an argon atmosphere using an Emitech K575X peltier in order to make the samples conductive. The microscopic analyses were performed at $5 \mathrm{kV}$ in a high resolution LEO1525 Karl Zeiss SEM. The X-ray diffraction patterns were acquired at room temperature on a PANalytical X'Pert Pro diffractometer using CuK $\alpha$ radiation (1.541 $\AA$ ), with a Nickel filter, a fixed $10 \mathrm{~mm}$ mask, a soller slit of $0.004 \AA$ and divergence and antiscatter slits of $1 / 4^{\circ}$ and $1 / 2^{\circ}$ respectively. The data was collected over 5 to $40^{\circ}$ angular range in $2 \theta$ in continuous scan mode using a step size of $0.05^{\circ}$ and a step time of $5 \mathrm{~s}$.

\subsection{OSN experimental procedure}

Each type of membrane was prepared at least two times from different dope solutions and tested a minimum of two times. The reported results are the mean values of these measurements. All filtration experiments were carried out at 10 bar using a cross-flow filtration system described previously in work from the Livingston group[33, 34]. The effective area of each membrane was 14 $\mathrm{cm}^{2}$, and 4 discs of each membrane were placed in an 8 cell cross-flow rig, which comprises of 2 parallel sets of 4 membranes in series. Permeability was calculated as given in Equation 1.

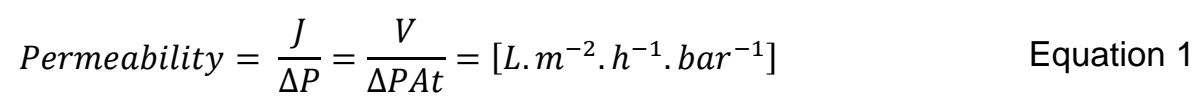

The permeance was obtained by measuring the solvent flux through the membrane $(J)$ and dividing this by the applied pressure across the membrane $(\Delta P)$. The flux was calculated by measuring the volume of solution $(V)$ that permeates through the membrane per unit area $(A)$ per unit time $(t)$. The model system for the solute rejection experiments comprised of a mixture of $1 \mathrm{~g} . \mathrm{L}^{-1}$ PS580 and PS1300 polystyrene marker as well as $0.1 \mathrm{~g} \cdot \mathrm{L}^{-1}$ of methyl styrene dimer solution in acetone. As given in Equation 2, the rejection $\left(R_{j}\right)$ of markers was found by measuring individual oligomer concentrations in the permeate $\left(C_{p, i}\right)$ and the feed $\left(C_{f, i}\right)$, respectively and calculating the ratio of the molecules retained by the membranes. 


$$
R_{j, i}=\left(1-\frac{C_{p, i}}{C_{f, i}}\right) \cdot 100=[\%]
$$

To determine membrane rejections samples of solution were taken from the feed and the permeate lines, leaving 24 hours of filtration prior to sampling to ensure the membrane performance had stabilised. Acetone was evaporated and the residue was re-dissolved in DMF. The HPLC analysis was based on the method previously reported by See-Toh et al [4]. Analysis of the polystyrene markers was undertaken using an Agilent HPLC system equipped with UV/Vis detector set at a wavelength of $264 \mathrm{~nm}$ and a Phenomenex C18 (300A, 250x4.6 mm) reverse phase column.

\subsection{Theoretical analysis of results (pore size probability function)}

Following other authors in this field we have adopted the log-normal probability density function to describe pore size. The density function has an advantage over the Gaussian distribution, being only defined for positive values of the pore diameter $\left(0<d_{p}<\infty\right)$ [35]. Most authors use the definition proposed by Belfort et al. [36] and latter reviewed and recommended by Zydney et al.[37]. The lognormal distribution is as follows:

$$
\begin{gathered}
f_{R}\left(d_{p}\right)=\frac{1}{d_{p} \sqrt{2 \pi b}} \exp \left[-\frac{\left(\left[\log \frac{d_{p}}{\bar{d}_{p}}\right]+\frac{b}{2}\right)^{2}}{2 b}\right] \\
b=\log \left[1+\left(\frac{\sigma}{\bar{d}_{p}}\right)^{2}\right]
\end{gathered}
$$

Equation 3

Equation 4

In order to estimate mean pore size $\left(\bar{d}_{\mathrm{p}}\right)$ and standard deviation $(\sigma)$ a fitting of experimental rejection data to the log normal distribution is performed. However, given that this approach lacks a physical basis, more recent research considers the underlying phenomena and utilises a hydrodynamic model of hindered solute transport in pores in estimating $\overline{\mathrm{d}}_{\mathrm{p}}$ and $\sigma$. In this work this approached is adopted. The starting point for this model is the extended Nernst-Planck equation for the transport of charged solutes; here we state the formulae and for further details we recommend Bowen and Welfoot (2002) [38, 39], Silva and Livingston (2006) [40] and Santos et al. (2006) [41].

From the Nernst-Planck equation an expression for rejection as a function of pore size can be obtained and is as follows:

$$
R_{i}=1-\frac{\phi\left(k_{c}+Y\right)}{1-\left[1-\phi\left(k_{c}+Y\right)\right] \exp (-P e)}
$$

Equation 5

The terms $\mathrm{Y}$ and $\mathrm{Pe}$ are, respectively, a dimensionless solute function (independent of solute concentration) and the Peclet number and can be calculated according to Equation 6 and Equation 7 .

$$
Y=\frac{32 \mu k_{d} D_{i, \infty} v}{R T d_{p}^{2}}
$$




$$
P e=\frac{\left(k_{c}+Y\right) d_{p}^{2}}{32 \mu k_{d} D_{i, \infty}}
$$

Where $\mu$ is viscosity, $D_{i, \infty}$ is the diffusion coefficients of the solutes in the bulk solvent, $v$ the molar volume of the solvent and $d_{p}$ is the pore diameter. The convective and diffusive hindrance factors $\left(k_{\mathrm{c}}\right.$ and $\left.k_{\mathrm{d}}\right)$ can be defined as:

$$
\begin{aligned}
k_{c} & =\frac{u}{u_{w}} \\
k_{d} & =\frac{D_{p}}{D_{i, \infty}}
\end{aligned}
$$

Equation 8

Equation 9

Where $u$ is the solute speed in the pore, $u_{\mathrm{w}}$ average solvent speed and $D_{\mathrm{p}}$ the solute diffusion coefficient in the pore. These hydrodynamic drag coefficients can be correlated with the solute to pore radii fraction, $\lambda$ (Equation 10Equation 11 Equation 12).

$$
\begin{gathered}
\lambda=\frac{d_{i}}{d_{p}} \\
k_{c}=A+B \lambda+C \lambda^{2}+D \lambda^{3} \\
k_{d}=E+F \lambda+G \lambda^{2}+H \lambda^{3}
\end{gathered}
$$

The values of the constants are presented in Table 2 [42] and are a result of detailed studies from Deen [43] and Bowen et al. [44] and [45].

Table 2: Values of the constants for equations 6 and 7 for $\lambda$ between 0 and 0.8 and $\lambda$ between 0.8 and 1 [42].

\begin{tabular}{|l|l|l|}
\hline Constant & $\mathbf{0}<\boldsymbol{\lambda} \leq \mathbf{0 . 8}$ & $\mathbf{0 . 8}<\boldsymbol{\lambda} \leq \mathbf{1}$ \\
\hline A & 1.0 & -6.830 \\
\hline B & 0.054 & 19.348 \\
\hline C & -0.988 & -12.518 \\
\hline D & 0.441 & 0 \\
\hline E & 1.0 & -0.105 \\
\hline F & -2.30 & 0.318 \\
\hline G & 1.154 & -0.213 \\
\hline H & 0.224 & 0 \\
\hline
\end{tabular}

These equations are valid for cylindrical pores with developing velocity profiles. When the pores are relatively narrow and long, the velocity profiles should fully developed, and $k_{\mathrm{c}}$ should be multiplied by $(2-\Phi)$ with $\Phi$ corresponding to the solubility equilibrium at the interfaces (Equation 13 ).

$$
\phi=(1-\lambda)^{2}
$$

The solute diameters $\left(d_{i}\right)$ were determined by the Stokes-Einstein equation: 


$$
d_{i}=\frac{k T}{3 \pi \mu_{0} D_{i, \infty}}
$$

Equation 14

The diffusion coefficients of the solutes in the bulk solvent were determined by the Wilke-Chang equation [46] (equation 15), where the solute molar volume $\left(V_{i}\right)$ at the boiling point was determined using a group contribution method as presented by Zhao et al. [47]. In cases where the pore diameters are significantly small and similar to the solvent diameter $\left(d_{p} \approx d_{\text {solv }}\right)$, pore viscosities need to be corrected since they are significantly higher than the bulk values $\left(\mu_{0}\right)$ [38] (Equation 16). This variation of viscosity was subject to the condition that $\eta=10 \eta_{0}$ when $d_{p}<0.46 \mathrm{~nm}$ [35].

$$
\begin{gathered}
D_{i, \infty}=\frac{1.173 \times 10^{-16}\left(\varphi M_{i}\right)^{0.5} T}{\mu_{0} V_{i}^{0.6}} \\
\frac{\mu}{\mu_{0}}=1+18\left(\frac{d_{\text {solv }}}{d_{p}}\right)-9\left(\frac{d_{\text {solv }}}{d_{p}}\right)^{2}
\end{gathered}
$$

For certain broad pore size distributions, it is expected that the 'tail' of large pores will affect the overall rejection due to the $d_{p}^{4}$ averaging of porewise rejection, $R_{i}^{\text {calc }}\left(d_{p}\right)$. It is suggested in literature that the distribution should be truncated to eliminate the effect of the 'tail' of large pores [35]. However, it is still desirable for the new distribution to be shaped like the log-normal distribution for those pores which contribute the overwhelming majority of the distribution. The total area under the predicted curve is computed using the trapezium rule. The new distribution function $\left(f_{R}{ }^{\prime}\right)$ is defined in Equation 17 and it is subject to the limiting case that $f_{R}{ }^{\prime}=f_{R}{ }^{\prime}$ as

$$
\begin{aligned}
\frac{f_{R}^{\prime}}{f_{R}} & =\frac{1}{\int_{0}^{d_{p_{\max }}} f_{R} d\left(d_{p}\right)} \\
R_{i}^{\text {calc }} & =\frac{\int_{0}^{d_{p_{\max }}} \frac{f_{R}^{\prime} d_{p}^{4} R_{i}}{\mu}\left(d_{p}\right)}{\int_{0}^{d_{p_{\max }}} \frac{f_{R}^{\prime} d_{p}^{4}}{\mu}\left(d_{p}\right)}
\end{aligned}
$$

$r_{\max } \rightarrow \infty$. The overall rejection is then calculated using the new distribution (Equation 18).

By introducing a truncated distribution a new parameter is required in any calculation, namely $d_{p_{\max }}$. Since there is limited available data on pore size distributions for nanofiltration membranes and current evidence suggests that pore diameter greater than twice the mean are uncommon, the upper limit set for the calculations was $d_{p_{\max }}=2 \bar{d}_{p}$.

\section{Results and Discussion}

\subsection{Chemical Modification as a Tool to Improve the Performance of Hybrid Polymer/MOF In-Situ Growth Membranes}

ATR-FTIR was used to detect the presence of CMA in the membranes. The spectra in Figure 2 show that the CMA has been grafted successfully onto the surfaces of the membranes M3 and M4. The broad peak between 2400 and $2700 \mathrm{~cm}^{-1}$ is demonstrative of the stretching of the carboxylic acid O- 
$\mathrm{H}$ bond in membranes UF(D)-CMA and ISG(D)-CMA, caused by the presence of the CMA in the membrane matrix. The characteristic peaks at 740, 1380 and $1450 \mathrm{~cm}^{-1}$ confirm the presence of HKUST-1[48] in membranes M2 and M4.

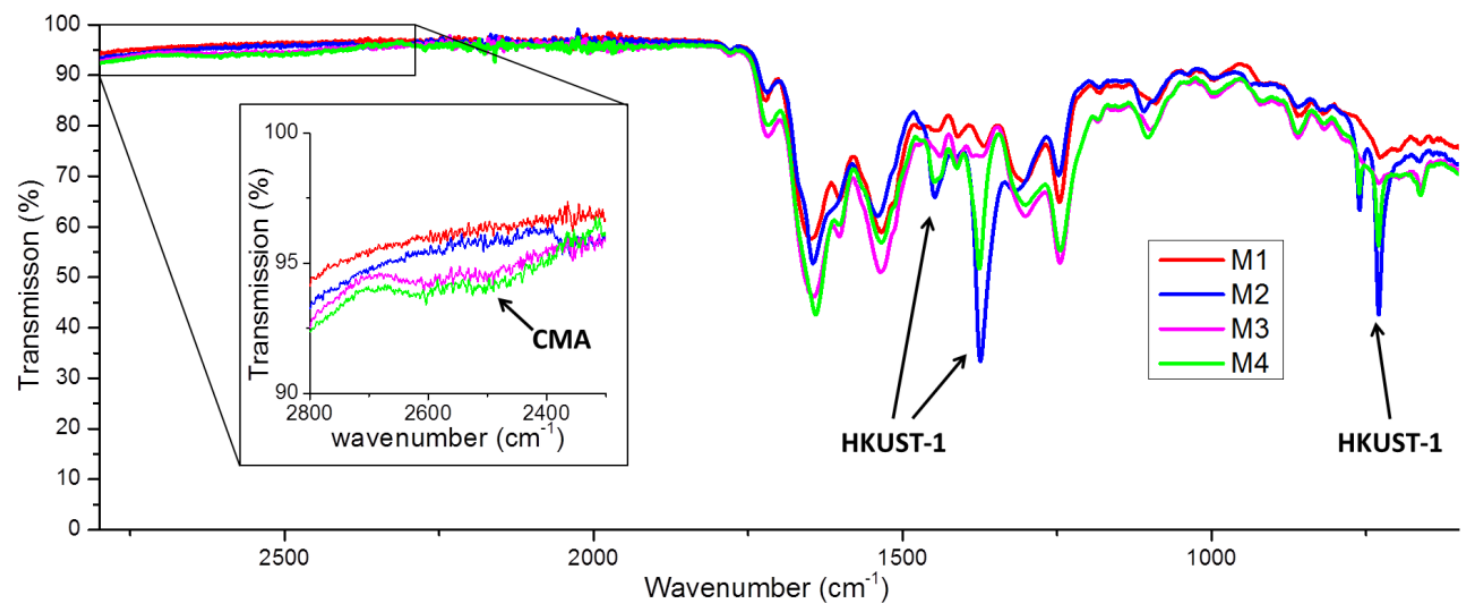

Figure 2: Chemical modification and MOF growth via ATR- FTIR spectra of the prepared polyimide membranes, the inset graph shows the broad peak associated with the addition of 1,2,4benzenetricarboxylic anhydride to the membrane structure.

The permeance data (see Figure 3 ) indicates that addition of the CMA has a tightening effect on the membrane. The average steady state permeance of membrane $M 1$ was $120 \mathrm{~L} \mathrm{~m}^{-2} \mathrm{~h}^{-1}$ bar $^{-1}$ while the average steady state permeance of membrane $\mathrm{M} 3$ is lower at $80 \mathrm{~L} \mathrm{~m}^{-2} \mathrm{~h}^{-1}$ bar $^{-1}$.

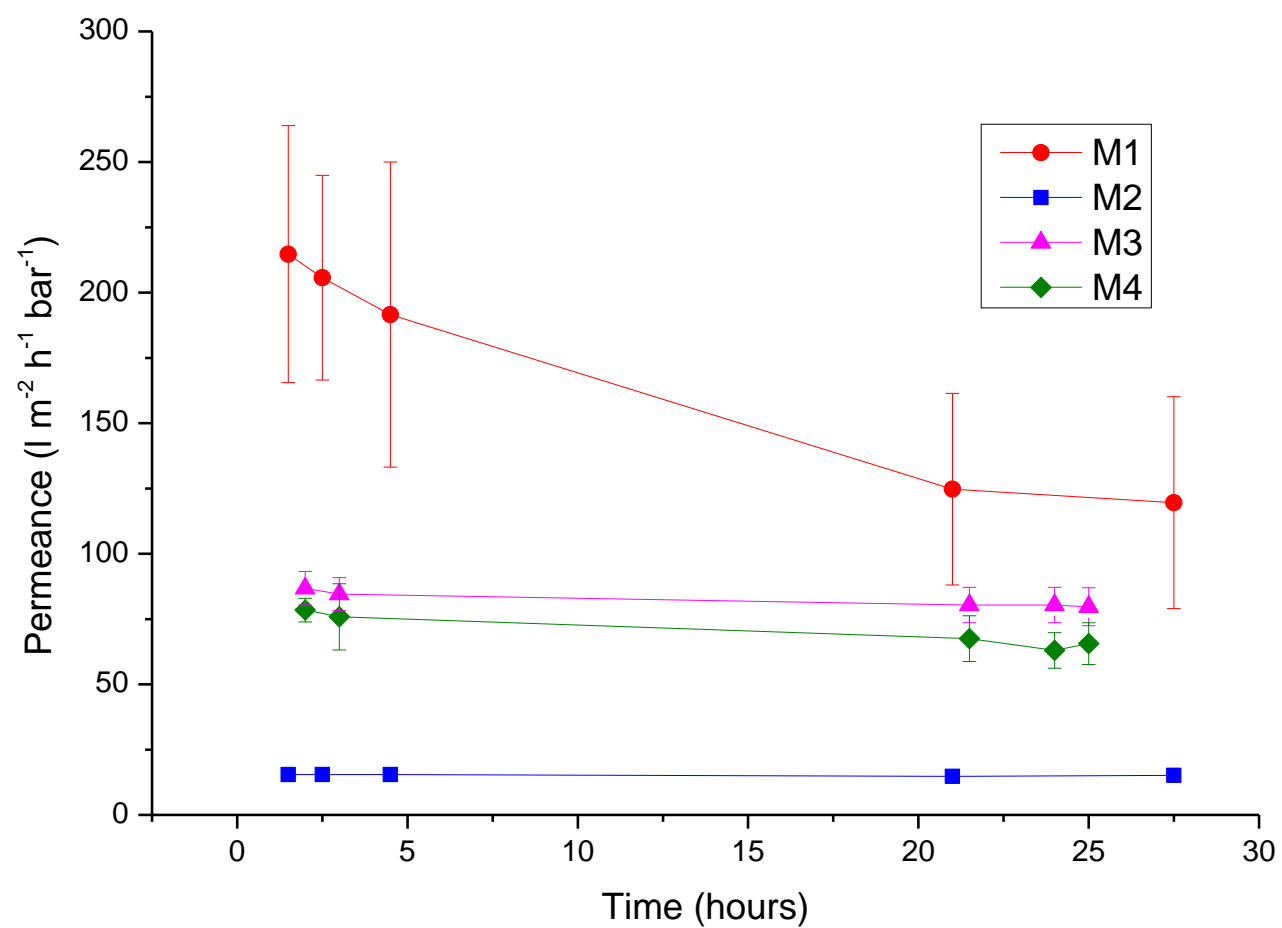

Figure 3: Permeance for acetone at 10 bar over time for the UF membranes (M1), chemically modified UF membrane (M3) and the ISG membranes (M2, M4). Mean data for each membrane plotted, error bars show are one standard deviation. 
The reduced permeance values of the MOF membranes, M2 and M4, indicate pore-filling has occurred and slowed solvent flow through the membrane pores. Pore-filling refers to the reduced porosity of membranes after the ISG process. In-situ growth of HKUST-1 within the pores of the UF membranes can hinder the permeance of solvent molecules by reducing the porosity of the membranes. The average permeance of membrane $M 2$ was $15 \mathrm{~L} \mathrm{~m}^{-2} \mathrm{~h}^{-1}$ bar $^{-1}$ at steady state, an almost tenfold reduction in permeance compared to membrane $M 1$, indicating that substantial pore-filling has occurred in this membrane. The average steady state permeance of membrane M4 was $66 \mathrm{~L} \mathrm{~m}^{-2} \mathrm{~h}^{-1}$ bar $^{-1}$ compared to $80 \mathrm{~L} \mathrm{~m}^{-2} \mathrm{~h}^{-1}$ bar $^{-1}$ for membrane $\mathrm{M} 3$. The reduction in permeance between membranes $\mathrm{M} 3$ and $\mathrm{M} 4$ was less than $18 \%$, while the reduction between membranes $\mathrm{M} 1$ and $\mathrm{M} 2$ was $87 \%$. This suggests that the addition of MOF to chemically modified polymer membranes results in less pore-filling, and points to less MOF being formed.

The addition of HKUST-1 to P84 ultrafiltration membranes is intended to modify the pores sufficiently so as to move the membrane molecular weight cut-off (MWCO) into the nanofiltration range $\left(200-1000 \mathrm{~g} \mathrm{~mol}^{-1}\right)$. Figure 4 shows the MWCO curves of each of membrane tested. The addition of the CMA to membrane M3 slightly increases the rejection of polystyrene markers as compared to the polystyrene rejection of the plain P84 membrane M1. As expected the addition of HKUST-1 to the P84 membranes increased the rejection of the polystyrene oligomers for each membrane. The addition of MOF to the unmodified P84 membrane (M1 to M2) increased the rejection of the membrane but not significantly enough to place the membrane MWCO into the nanofiltration range. The chemically modified MOF membrane M4 showed an increase in rejection above the base P84 membrane, M1 and also the chemically modified membrane, M3. The MWCO of membrane M4 is within the nanofiltration range at $794 \mathrm{~g} \mathrm{~mol}^{-1}$. Membrane M4 outperforms membrane $\mathrm{M} 2$ in both rejection and permeance. The use of the chemical modification agent appears to avoid the defects associated with uncontrolled growth of MOF found with membrane M2.

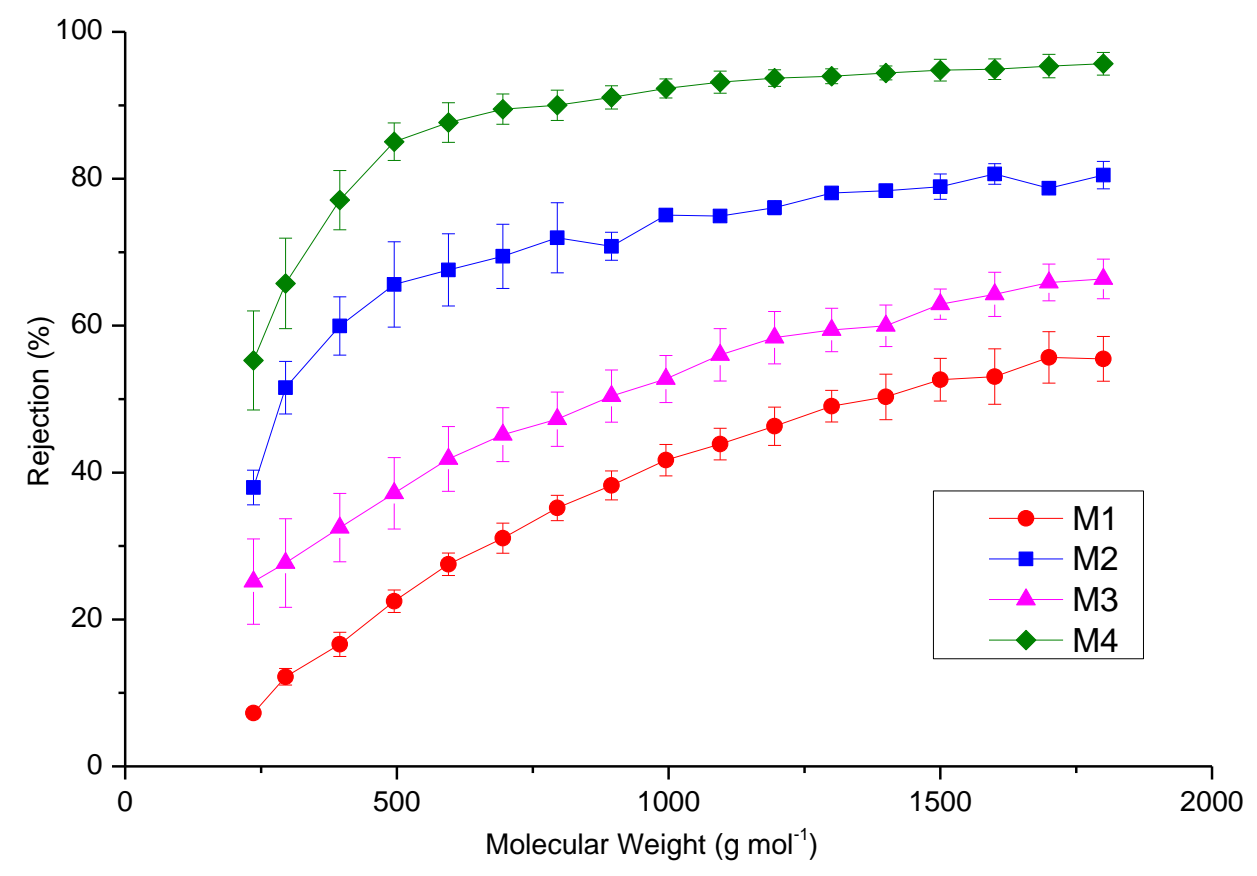


Figure 4: MWCO curves for the UF membranes (M1), the chemically modified UF membrane (M3) and the ISG membranes (M2, M4) tested at 10 bar with polystyrene in acetone solvent. Mean data for each membrane plotted, error bars show one standard deviation.

While membrane M4 displayed the highest solute retentions of all the membranes, physical evidence of improved growth characteristics of MOF in the membrane is less conclusive. Addition of MOF material to a membrane should result in an increase in the weight of the membrane. The mass, density and thickness of the membranes can be found in Table 3.

Table 3: Thickness, the specific mass, and density of the membranes fabricated in this study

\begin{tabular}{|c|c|c|c|}
\hline Membrane & $\begin{array}{l}\text { Thickness } \\
\qquad(\mu \mathrm{m})\end{array}$ & $\begin{array}{c}\text { Specific Mass } \\
\left(\mathrm{g} \cdot \mathrm{m}^{-2}\right)\end{array}$ & $\begin{array}{l}\text { Density } \\
\left(\mathrm{g} \cdot \mathrm{cm}^{-3}\right)\end{array}$ \\
\hline M1 & $67 \pm 1$ & $43 \pm 3$ & $0.64 \pm 0.05$ \\
\hline $\mathrm{M} 2$ & $65 \pm 3$ & $63 \pm 2$ & $0.97 \pm 0.06$ \\
\hline M3 & $91 \pm 1$ & $55 \pm 0$ & $0.6 \pm 0.01$ \\
\hline M4 & $78 \pm 2$ & $53 \pm 5$ & $0.68 \pm 0.05$ \\
\hline
\end{tabular}

The densities of membranes M2 and M4 are higher than the non-MOF membranes (M1 and M2 respectively) from which they are formed. However the increase in density is lower from M3 to M4 than M1 to M4, indicating that the amount of MOF grown in M4 is lower than M2. Using the calculation for pore filling percentage devised in previous work[14] to calculate the volume of UF membrane pores filled with HKUST-1 material the pore filling by HKUST-1 in M2 is $52 \%$, while the pore filling in M4 is only $12 \%$. The difference in pore filling in the membranes is likely to have contributed to the lower permeance performance of $\mathrm{M} 2$ as compared to M4. 


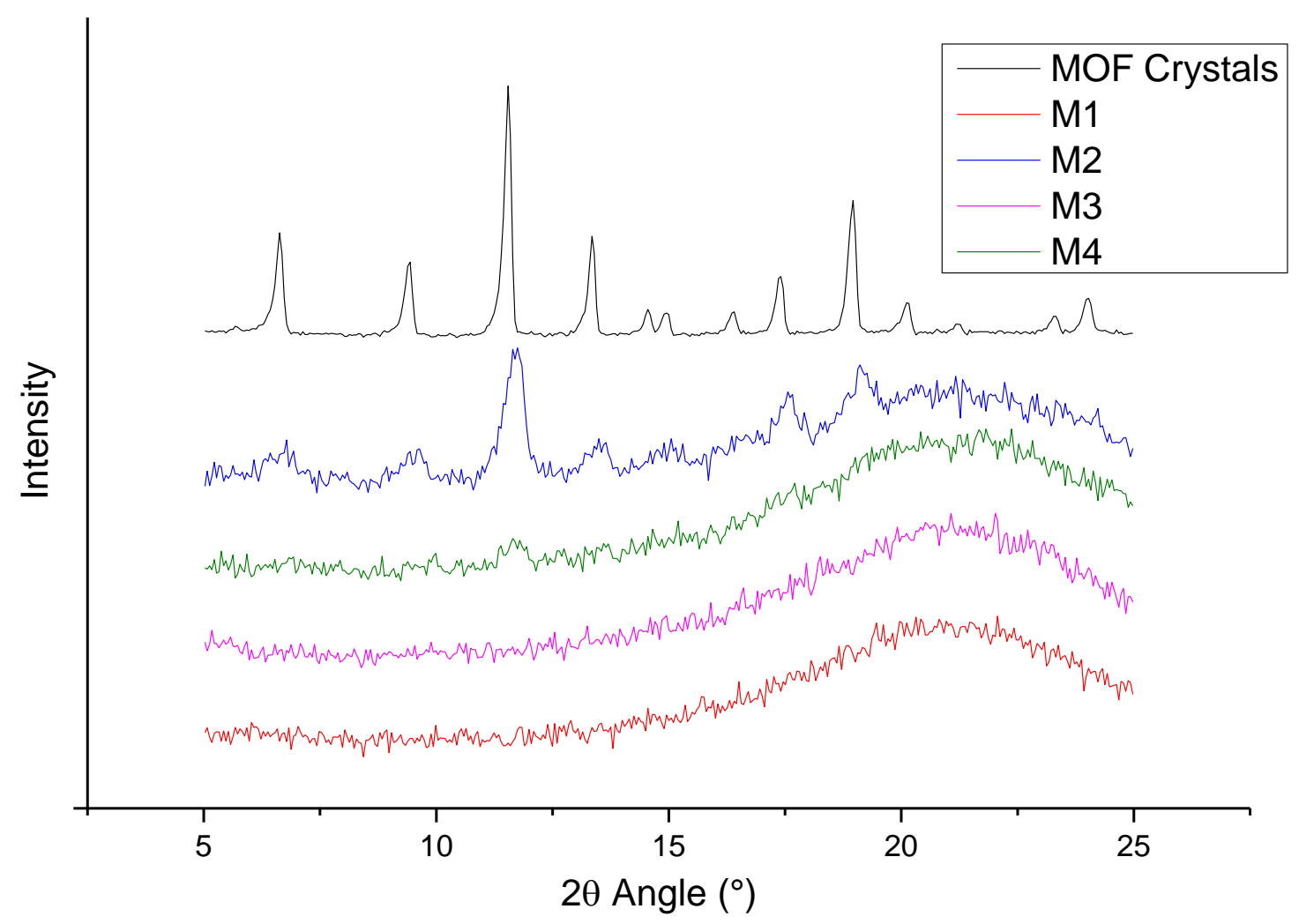

Figure 5: XRD data for the membranes alongside the data for pure HKUST-1 crystals

The presence of crystalline HKUST-1 material in hybrid polymer/MOF membranes was confirmed using X-ray diffraction (XRD). Figure 5 shows the XRD data for each of the membranes, compared to the XRD of pure HKUST-1 crystals. The non-MOF membranes (M1 \& M3) show no sign of MOF crystallinity, and they have very similar XRD readings to each other. The characteristic peaks of HKUST-1 occur at $2 \theta$ angles $6.6,9.4,11.5$ and 13.3, the membrane M2 shows large peaks at these values. Membrane M4 only has one small peak at 13.3, confirming that the amount of HKUST-1 grown in the chemically modified membrane is less than the membrane without CMA addition.

Figure 6 shows the percentage of copper measured throughout the cross-sections of the MOF containing membranes. The EDX data suggests that atomically, between $3 \%$ and $8 \%$ of membrane M2 is copper, confirming the presence of HKUST-1 material in these membranes. EDX data for the membrane M4 shows that the concentration of copper throughout the membrane ranges from $0 \%$ to just below $3 \%$, once again indicating that this membrane actually contains less HKUST-1 material than membrane $\mathrm{M} 2$, contributing to the improved permeance performance of membrane M4. 


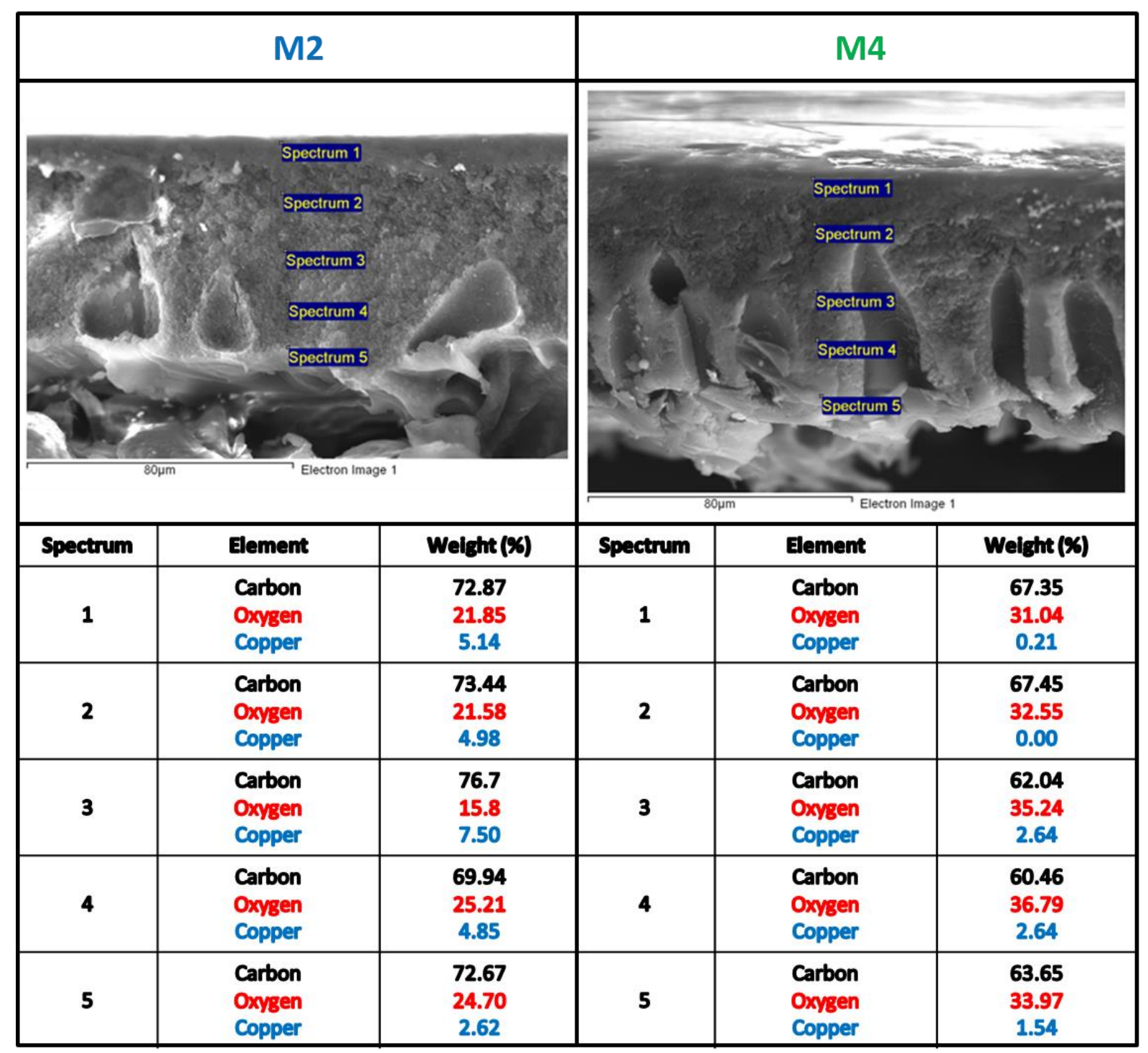

Figure 6: (left) SEM image of M2 used to find point spectrum EDX data (right) SEM image of M4 used to find point spectrum EDX data. The data in each table shows EDX elemental analysis for membranes with each spectrum point corresponds to the points indicated in the SEM images.

Despite the rejection performance of the chemically modified membrane M4 being an improvement on the original ISG membrane M2, the evidence from the XRD data, mass and density measurements and SEM/EDX data suggests that the membrane actually contains substantially less HKUST-1 material. Therefore the addition of CMA does not cause an increase in growth of MOF within the membranes. However the MOF material that does grow may have better adhesion to the polymer surface, and the HKUST at the surface of membrane M4 could contain fewer defects than membrane M2. The ATR-FTIR spectrum (Figure 2) of M4 shows that the membrane has a strong HKUST-1 signal at the membrane surface, supporting the hypothesis that M4 contains a very thin, low defect, MOF layer at the membrane surface. One theory on how this could occur is that the chemical modification agent allows for the formation of a highly ordered MOF film at the membrane surface, which blocks the diffusion of copper nitrate and/or benzene tricarboxylic acid further into the membrane. This would result in the formation of a thin MOF film at the membrane surface, which would also explain the high permeance value measured for the membrane M4. The theory is 
supported by the SEM/EDX of membrane M4 in Figure 6. Spectrum 2, in the spongy layer of the polymer membrane contains no copper; while the other spectrums contain observable quantities.

\subsection{Theoretical Pore Size Analysis}

The addition of HKUST-1 to polymer supports via in-situ growth has been shown to increase the retention of polystyrene solutes, as well as altering the shape of the MWCO curves. Log-normal probability functions have been calculated in order to estimate how the addition of MOF to the polymer membranes has altered the theoretical pore size distributions. Figure 7 shows the probability function curves for the four membranes.

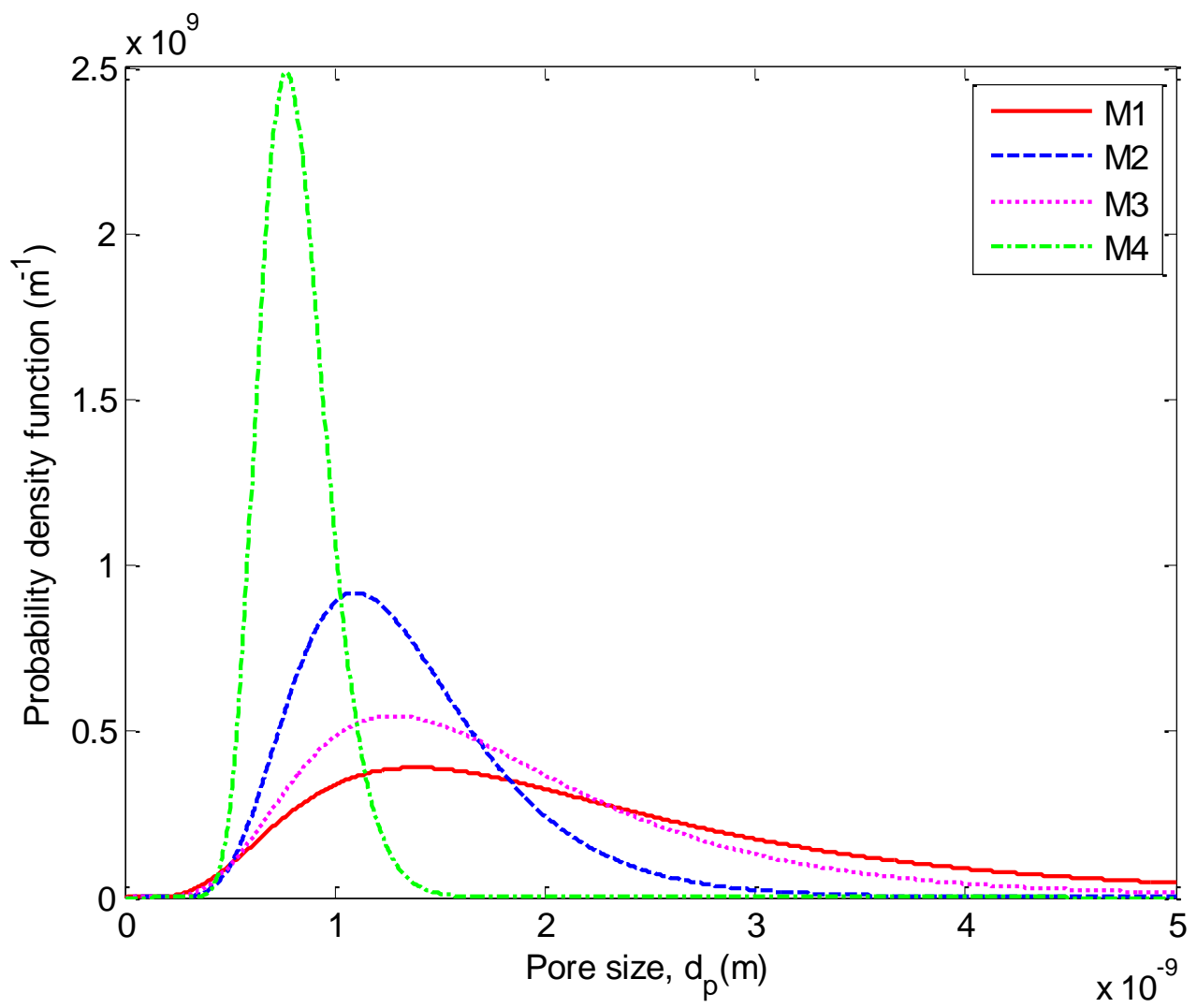

Figure 7: Probability density function curves for pore sizes $\left(d_{\mathrm{p}}\right)$ of membranes M1 to M4

The polymer membranes M1 and M3 show broad plots, with significant probability of finding pores above $3 \mathrm{~nm}$. Alternatively, the hybrid membranes $\mathrm{M} 2$ and $\mathrm{M} 4$ have much narrower distributions than the polymer membranes. These curves suggest that the addition of HKUST-1 not only increases the retention of solutes by reducing the average pore size of polymer membranes, but also narrows the pore size distribution via the addition of a regular porous structure. The calculated theoretical mean pore size and standard deviation of the membranes are shown in Table 4.

The hybrid MOF membranes M2 and M4 have the lowest calculated mean pore sizes, which is to be expected, as these membranes have the highest solute retentions. The combination of CMA addition and HKUST-1 addition of polymer UF membranes transforms the average pore size from $2.43 \mathrm{~nm}$ to $0.82 \mathrm{~nm}$. The mean pore size of membrane M4 is lower than the pore diameter of HKUST-1. This could be due to the presence of smaller pores in the polymer membrane. The log- 
normal probability functions of the membranes show that all the membranes, including the polymer membranes have a reasonable probability of having pores below $0.6 \mathrm{~nm}$.

Table 4: Theoretical mean pore size, standard deviation and the coefficient of variation of the membranes M1 to M4

\begin{tabular}{|l|c|c|c|}
\hline Membrane & $\begin{array}{c}\text { Mean Pore Diameter } \\
(\mathbf{n m})\end{array}$ & $\begin{array}{c}\text { Standard Deviation } \\
(\mathbf{n m})\end{array}$ & Coefficient of Variation \\
\hline M1 & 2.43 & \pm 1.63 & 0.67 \\
\hline M2 & 1.35 & \pm 0.52 & 0.38 \\
\hline M3 & 1.87 & \pm 1.00 & 0.53 \\
\hline M4 & 0.82 & \pm 0.17 & 0.21 \\
\hline
\end{tabular}

Membrane M4 has the lowest standard deviation of all the membranes, which is in accordance with the shape of the membrane's probability function. Further analysis shows that this reduction in standard deviation is not just due to the lower mean pore size, as membrane M4 also has a much lower coefficient of variation than the other membranes.

Analysis of the theoretical pore size distribution of the hybrid membranes shows that the addition of MOF appears to narrow the pore size distribution of the polymer membranes. The low standard deviation and coefficient of variation of membrane M4 shows that the addition of CMA followed by formation of HKUST-1 in P84 UF membranes can create hybrid membranes with more regular porous structures in the selective layer of the membrane.

\subsection{The Quest for the Perfect MOF Membrane}

Using chemical modification agents to reduce defects during ISG membrane formation led to the creation of a hybrid membrane with a MWCO in the nanofiltration range, with a narrower pore size distribution. The physical evidence (ATR-FTIR, mass and density measurements and SEM-EDX) suggests that membrane M4 has a thin layer of HKUST-1 embedded in the selective layer of the polymer membrane. The high solute retentions and the high permeance values achieved with the membrane also suggest that a low defect, thin MOF film has been formed within the selective layer of the hybrid membrane. This section evaluates how close to perfection membrane M4 has come.

A defect free HKUST-1 film would have a single pore size of $0.9 \mathrm{~nm}$ (the $0.6 \mathrm{~nm}$ pores which connect the cages can be ignored for separation purposes). The pores consist of straight, circular channels throughout the MOF structure in all three directions. The size of each cage unit of HKUST-1 is $1 \mathrm{~nm}$, therefore a defect free HKUST film would have a porosity of 0.64 . The structure of HKUST-1 as viewed from above can be seen in Figure 8. 


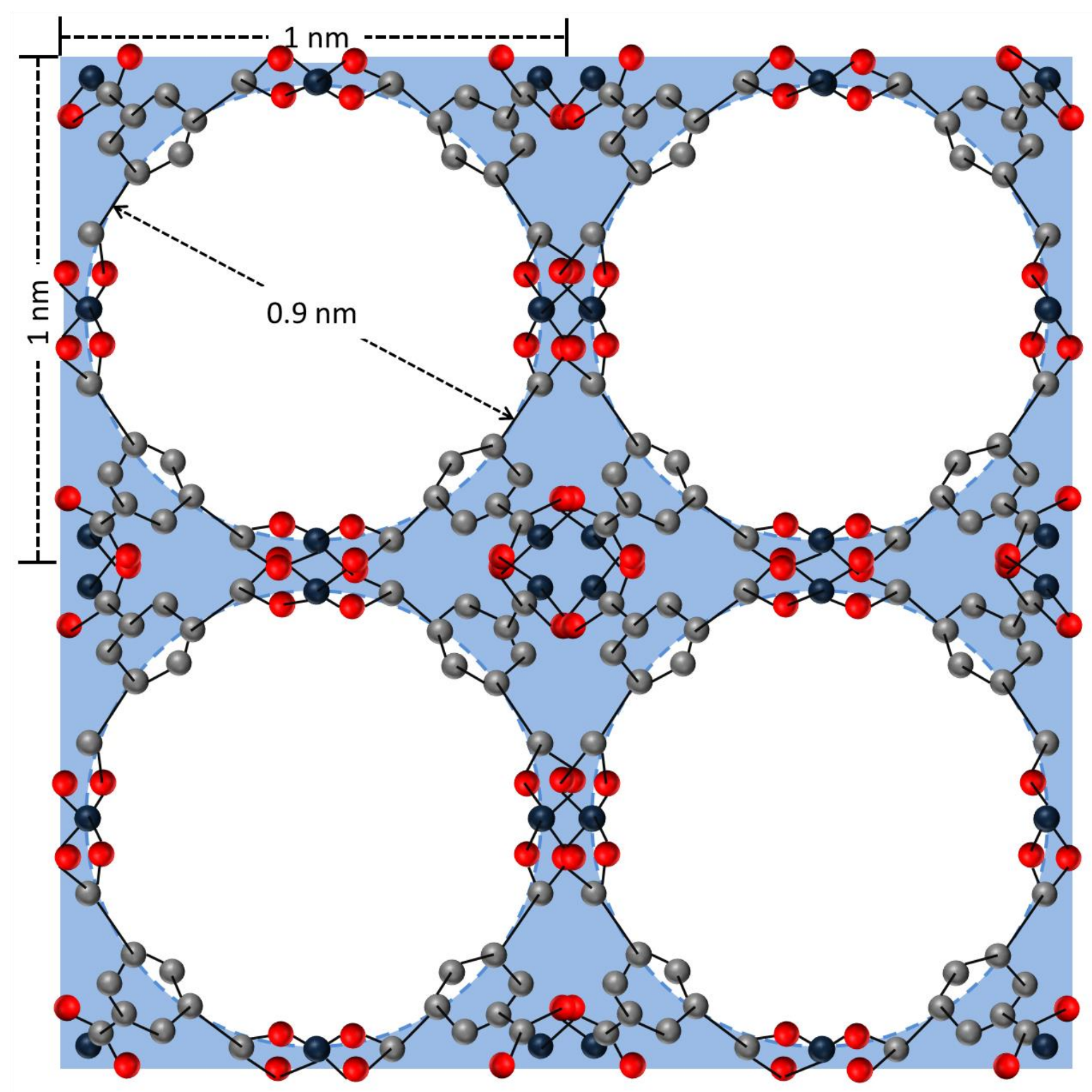

Figure 8: Structure of HKUST-1. The grey, red and blue spheres represent carbon, oxygen and copper atoms respectively. The MOF is viewed from above looking straight down through the spherical pores.

Using the pore flow transport model, with the pore viscosity adjustment as described by Bowen \& Welfoot[49] the permeance of acetone through HKUST-1 films of various thicknesses can be predicted. These predicted permeance values, along with the physical evidence obtained from membrane characterisation methods can give some clues as to the thickness of the HKUST-1 film in membrane M4. Values for permeance in $\mathrm{L} \mathrm{m}^{-2} \mathrm{~h}^{-1} \mathrm{bar}^{-1}$ are given in Table 5 for pure, defect free HKUST-1 films. The permeance of real hybrid membranes containing HKUST-1 would vary depending on the amount of defects in the film and the porosity of the polymer membrane. 
Table 5: Relationship between MOF (HKUST-1) film thickness and theoretical pure acetone permeance

\begin{tabular}{|c|c|}
\hline $\begin{array}{c}\text { MOF Film Thickness } \\
(\boldsymbol{\mu m})\end{array}$ & $\begin{array}{c}\text { Calculated Permeance } \\
\left(\mathbf{L} \mathbf{~}^{-\mathbf{2}} \mathbf{h}^{-\mathbf{1}} \mathbf{b a r}^{-\mathbf{1})}\right.\end{array}$ \\
\hline 100 & 0.18 \\
\hline 50 & 0.35 \\
\hline 4 & 4.4 \\
\hline 1 & 18 \\
\hline $0.1(100 \mathrm{~nm})$ & 180 \\
\hline $0.035(35 \mathrm{~nm})$ & 500 \\
\hline $0.001(1 \mathrm{~nm})$ & 18000 \\
\hline
\end{tabular}

SEM-EDX analysis has shown that the typical film thickness of ISG membranes formed via uncontrolled growth, such as membrane $\mathrm{M} 2$, is $100 \mu \mathrm{m}$, i.e. the entire thickness of the polymer membrane. If every pore of a UF membrane, including the macrovoids, were filled with HKUST-1, the permeance observed for a defect-free hybrid ISG membrane would be would be $0.18 \mathrm{~L} \mathrm{~m}^{-2} \mathrm{~h}^{-1}$, an extremely low permeance. This value would be reduced if the original porosity of the UF membrane was low, but much higher if HKUST-1 was not able to completely fill the macrovoid pores. Typically ISG membranes have permeances between 15 and $18 \mathrm{~L} \mathrm{~m}^{-2} \mathrm{~h}^{-1}$ bar[14, 50]. These permeances are relative high, indicating that the membranes are not defect free. SEM images of MOF-TFCs indicate that the thicknesses of the MOF films formed via interfacial synthesis are between 1 and $4 \mu \mathrm{m}$ [50]. For films this thick a permeance of between 4.4 and $18 \mathrm{~L} \mathrm{~m}^{-2} \mathrm{~h}^{-1} \mathrm{bar}^{-1}$ is predicted. The permeance of membrane $M 4,66 \mathrm{~L} \mathrm{~m}^{-2} \mathrm{~h}^{-1}$, is higher than this, suggesting that the thickness of the MOF film in chemically modified ISG membranes is thinner than those achievable using interfacial synthesis methodologies. The thinnest possible HKUST-1 film would be $1 \mathrm{~nm}$, which corresponds to the width of a single HKUST-1 cage. A MOF film of this thickness may not be mechanically stable under high pressures; however films with thicknesses up to 100 HKUST-1 cage units can have permeances of up to $180 \mathrm{~L} \mathrm{~m}^{-2} \mathrm{~h}^{-1} \mathrm{bar}^{-1}$. Though it is likely in reality that for extremely thin MOF films the permeance of hybrid membranes would be determined by the permeability of the polymer support membrane.

Membrane characterisation, as well as the calculated permeances found in Table 3, suggests that the HKUST-1 film present in membrane M4 has a thickness likely well below $1 \mu \mathrm{m}$. Membrane M4 has a MWCO in the nanofiltration range, however the rejection of polystyrene solutes never reaches $100 \%$, thus it is likely not defect free.

The rejection of a defect free HKUST-1 membrane with a single pore size of $0.9 \mathrm{~nm}$ was simulated for polystyrene markers in acetone. This theoretical rejection of an ideal HKUST-1 film was compared to the rejection achieved using membrane M4, and the result can be seen in Figure 9. It can be seen that the rejection of polystyrene reaches $100 \%$ at $595 \mathrm{~g} \mathrm{~mol}^{-1}$ whereas the retention of polystyrene markers never reaches $100 \%$ with membrane M4. This is an indication that the HKUST-1 film formed in the hybrid membrane is not completely defect free. The calculated MWCO for the ideal HKUST-1 membrane is $422 \mathrm{~g} \mathrm{~mol}^{-1}$, lower than the observed MWCO of membrane M4, $794 \mathrm{~g} \mathrm{~mol}^{-1}$. The theoretical rejection of the ideal HKUST-1 film is higher for every polystyrene oligomer except for the marker with a mass equal to $236 \mathrm{~g} \mathrm{~mol}^{-1}$. 


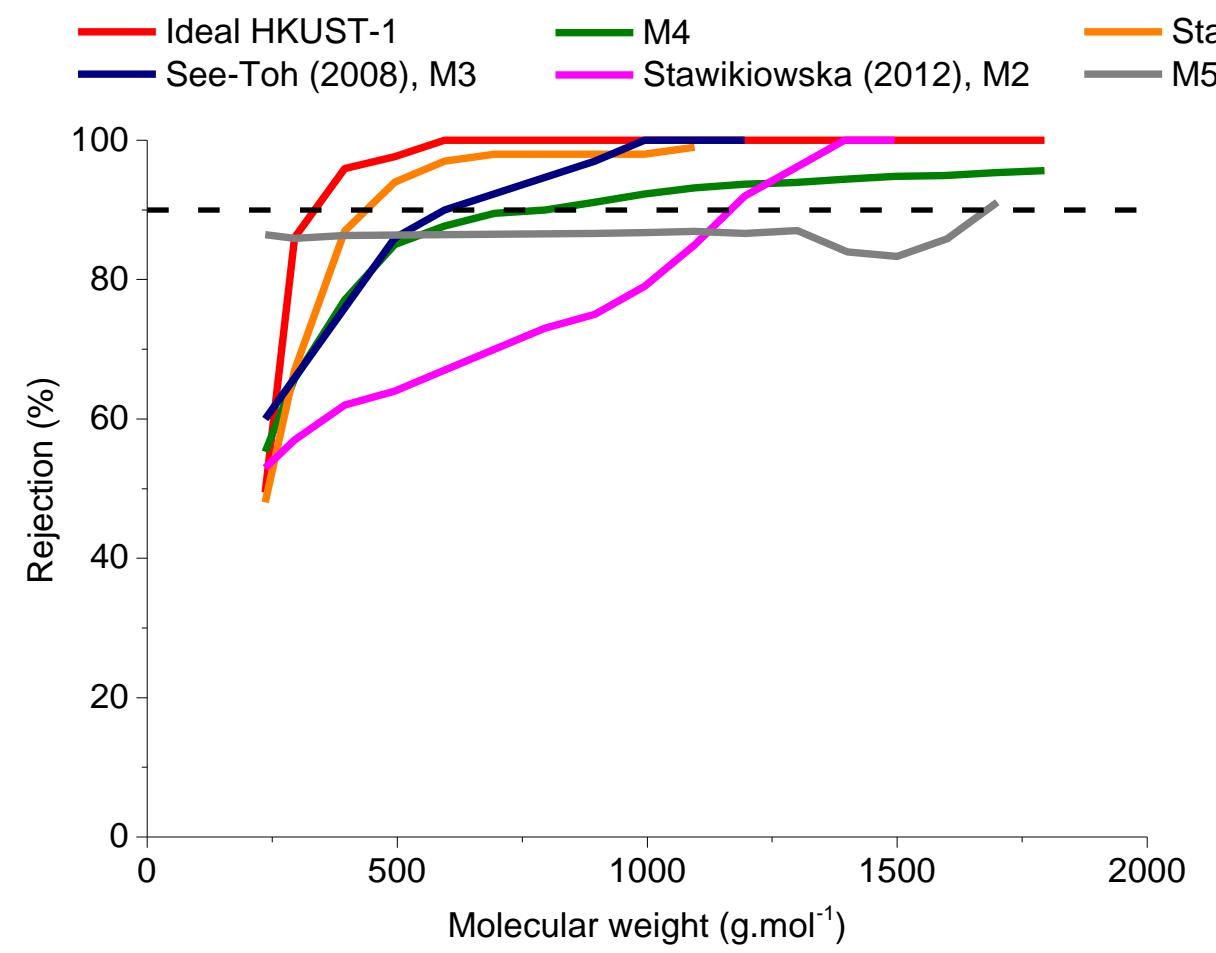

Figure 9: MWCO curves for hybrid membrane M4, Membranes M3 and M2, P84 nanofiltration membranes made using 2:1 ratios of DMF:Dioxane by See-Toh et al..[4] and Stawikowsa et al.[31] respectively, commercial membrane Starmem 240[51], polyimide nanofiltration membrane M5 and an ideal 'perfect' HKUST-1 film

The theoretical MWCO curve for the ideal HKUST-1 film is much 'sharper' than the observed curve for membrane M4. This suggests that the separation between closely related molecules would be greater for the ideal HKUST-1 film than for membrane M4. The improved sharpness of the theoretical MWCO curve for the ideal HKUST-1 membrane is likely due to the fact that the membrane has a single pore size and not a pore size distribution as observed with the current hybrid membranes.

The performance of the chemically modified ISG membrane, M4, is also compared to polyimide P84 nanofiltration membranes. Rejection data for the commercially available Starmem 240 and membranes previously produced by the Livingston group and reported by See-Toh et al.[4] and Stawikowsa et al.[31]. The membranes produced by See-Toh et al. and Stawikowsa et al. were both fabricated using dope solutions containing a 2:1 ratio of DMF:Dioxane as the solvent mixture. By altering the solvent mixtures within the dope solutions the solute retentions of the membranes could be altered. Membrane M5, produced for this study, was fabricated using a dope solution solvent ratio of 1:1. With a higher percentage of dioxane in the dope solution, the solute retention should be as high as or higher than these previously reported membranes.

Membranes M4 \& M5 was tested in acetone, while the other nanofiltration membranes were tested in toluene. While a direct comparison between results in different solvents should be taken with a pinch of salt the similarities between the performance of Membrane M5 in acetone and the other nanofiltration membranes in toluene indicate that general observation are true no matter the choice of solvent. 
The solute retention of the Starmem 240 membrane was found to almost exactly mirror the rejection achievable using an ideal HKUST-1 membrane. However, as Table 6 shows the permeance of this membrane is two orders of magnitude lower than the potential permeance for a 1 micron HKUST-1 film (180 $\mathrm{L} \mathrm{m}^{-2} \mathrm{~h}^{-1}$ bar $^{-1}$ as compared to $2 \mathrm{~L} \mathrm{~m}^{-2} \mathrm{~h}^{-1}$ bar $^{-1}$ ).

See-Toh's membrane has similar solute retentions as membrane M4, up to $495 \mathrm{~g} \mathrm{~mol}^{-1}$, after which the P84 nanofiltration membrane outperforms the hybrid ISG membrane. Stawikowska's membrane has much lower solute retentions than M4 until $1295 \mathrm{~g} \mathrm{~mol}^{-1}$. This shows that ISG membrane fabrication can produce membranes with solute rejections in the nanofiltration range. However, as Table 6 shows, the permeance of the hybrid ISG MOF membrane is over 6 times higher than these P84 nanofiltration membranes. The higher permeance is likely due to the open, porous nature of the ultrafiltration base membrane. The higher permeances achievable for ISG membranes offer a clear advantage over pure polymer nanofiltration membranes.

The membranes produced by See-Toh et al. and Stawikowska et al. have similar rejection curves to M4, however as the membranes were tested in a different solvent, toluene rather than acetone. M5 was tested in the same acetone polystyrene solution as M4. The permeance of M5 is of the same order of magnitude as the other polymer nanofiltration membranes, suggesting that the use of acetone as a solvent is not responsible for the improved permeance performance of M4 over the polymer nanofiltration membranes.

Table 6: Permeance of chemically modifed ISG M4 compared to pure polymer nanofiltration membranes with similar solute retentions

\begin{tabular}{|c|c|}
\hline Membrane & $\begin{array}{c}\text { Permeance } \\
\left(\mathbf{L ~ m}^{-\mathbf{2}} \mathbf{~}^{-\mathbf{1}} \text { bar }^{-\mathbf{1})}\right.\end{array}$ \\
\hline M4 & 66 \\
\hline Starmem 240 & $2.0[51]$ \\
\hline See-Toh (2008), M3 & $9.9[4]$ \\
\hline Stawikowska (2012), M2 & $9.0[31]$ \\
\hline M5 & 4.9 \\
\hline
\end{tabular}

Previous work by See-Toh et al. and Stawikowska et al. has measured the pore size distribution of nanofiltration membranes both theoretically and experimentally. See-Toh calculates that the average pore size of the membrane M3 is $1.09 \mathrm{~nm}$ with a standard deviation at $0.09 \mathrm{~nm}$. The standard deviations of the various 2:1 DMF:Dioxane membranes produced in See-Toh's work is highly variable, with standard deviations up to $0.61 \mathrm{~nm}$ for a given average pore size of $1.01 \mathrm{~nm}$. Stawikowska et al. measured the pore size distribution of 3 polymer nanofiltration membranes directly using osmium nanoparticles. The average pore size of the membrane $\mathrm{M} 2$ in this work was $0.98 \mathrm{~nm}$, higher than the chemically modified ISG membrane, with a very similar standard deviation of $0.16 \mathrm{~nm}$. The numbers from both these studies suggest that the pore size distributions of chemical modified ISG membranes are very similar to polymer nanofiltration membranes with similar rejections. With further improvement of the ISG fabrication process the pore size distribution of MOF hybrid membranes might be narrowed, leading to increased solute retentions as shown in Figure 9.

\section{Conclusions}


This paper demonstrates that the performance of hybrid polymer/MOF ISG membranes can be improved through the use of a chemical modification agent (CMA) to encourage direct MOF growth on the pore walls of the polymer membrane. In this work 1,2,4-benzenetricarboxylic anhydride was used to introduce carboxylate functional groups to dense polyimide P84 polymer membrane supports in order to improve the chemical adhesion of HKUST-1 to the membranes. Comparisons of the solute rejection curves indicate that chemical modification and HKUST-1 growth have a positive effect on membrane performance. Membrane M4 displayed high rejections with a molecular weight cut-off of $794 \mathrm{~g} \mathrm{~mol}^{-1}$, and high permeances.

The ISG membranes were characterised using a number of analytical methodologies, include ATRFTIR, SEM/EDX, XRD and mass and density analysis. These membrane characterisation methodologies indicated that the mass of HKUST-1 grown in membrane M4 was lower than in the non-chemically modified ISG membrane, M2. Despite the lower mass of HKUST-1 growth, the high solute retentions and the ATR-FTIR data suggests that the HKUST-1 had good adhesion to the membrane surface, and forming a low-defect, thin film on the surface of the membrane.

Analysis of the solute retention results show that ISG produces membranes with narrower pore size distributions than the polymer membranes from which they are formed. Theoretical calculations of the permeance of defect free HKUST-1 films show that membrane M4 is likely to consist of a thin MOF film within, or on top of the polymer UF support membrane. However the MWCO curve shows that the MOF film is not completely defect free, and improvements could be made in both the overall solute retentions and the sharpness of the MWCO curve.

\section{Acknowledgements}

The authors would like to acknowledge financial support provided by the EPSRC, UK - DTA awards EP/P504953/1; EP/P505550/1; Platform Grant award EP/J014974/1 and the financial support from the Pharmacat Consortium.

\section{References}

[1] P. Marchetti, M.F.J. Solomon, G. Szekely, A.G. Livingston, Molecular Separation with Organic Solvent Nanofiltration: A Critical Review, Chemical Reviews, (2014).

[2] P. Vandezande, L.E.M. Gevers, I.F.J. Vankelecom, Solvent resistant nanofiltration: separating on a molecular level, Chemical Society Reviews, 37 (2008) 365-405.

[3] A.K. Hołda, I.F.J. Vankelecom, Understanding and guiding the phase inversion process for synthesis of solvent resistant nanofiltration membranes, Journal of Applied Polymer Science, 132 (2015) 42130.

[4] Y.H. See-Toh, M. Silva, A. Livingston, Controlling molecular weight cut-off curves for highly solvent stable organic solvent nanofiltration (OSN) membranes, Journal of Membrane Science, 324 (2008) 220-232.

[5] S. Basu, A. Cano-Odena, I.F.J. Vankelecom, Asymmetric Matrimid ${ }^{\oplus} /\left[\mathrm{Cu}_{3}(\mathrm{BTC})_{2}\right]$ mixed-matrix membranes for gas separations, Journal of Membrane Science, 362 (2010) 478-487.

[6] S. Basu, A. Cano-Odena, I.F.J. Vankelecom, MOF-containing mixed-matrix membranes for $\mathrm{CO}_{2} / \mathrm{CH}_{4}$ and $\mathrm{CO}_{2} / \mathrm{N}_{2}$ binary gas mixture separations, Separation and Purification Technology, 81 (2011) 31-40.

[7] M.A. Aroon, A.F. Ismail, T. Matsuura, M.M. Montazer-Rahmati, Performance studies of mixed matrix membranes for gas separation: A review, Separation and Purification Technology, 75 (2010) 229-242. 
[8] J. Hu, H. Cai, H. Ren, Y. Wei, Z. Xu, H. Liu, Y. Hu, Mixed-Matrix Membrane Hollow Fibers of $\mathrm{Cu}_{3}(\mathrm{BTC})_{2} \mathrm{MOF}$ and Polyimide for Gas Separation and Adsorption, Industrial Chemical Engineering Research, 49 (2010) 12605-12612.

[9] E.V. Perez, K.J.B. Jr., J.P. Ferraris, I.H. Musselman, Mixed-matrix membranes containing MOF-5 for gas separations, Journal of Membrane Science, 328 (2009) 165-173.

[10] Y. Zhang, I.H. Musselman, J.P. Ferraris, K.J.B. Jr., Gas permeability properties of Matrimid ${ }^{\circledR}$ membranes containing the metal-organic framework CU-BPY-HFS, Journal of Membrane Science, 313 (2008) 170-181.

[11] A. Car, C. Stropnik, K.-V. Peinemann, Hybrid membrane materials with different metalorganicframeworks (MOFs) for gas separation, Desalination, 200 (2006) 424-426.

[12] R. Adams, C. Carson, J. Ward, R. Tannenbaum, W. Koros, Metal organic framework mixed matrix membranes for gas separations, Microporous and Mesoporous Materials, 131 (2010) 13-20.

[13] S. Basu, M. Maes, A. Cano-Odena, L. Alaerts, D.E.D. Vos, I.F.J. Vankelecom, Solvent resistant nanofiltration (SRNF) membranes based on metal-organic frameworks, Journal of Membrane Science, 344 (2009) 190-198.

[14] J. Campbell, G. Szekely, R.P. Davies, D.C. Braddock, A.G. Livingston, Fabrication of hybrid polymer/metal organic framework membranes: mixed matrix membranes versus in situ growth, Journal of Materials Chemistry A, 2 (2014) 9260-9271.

[15] L. Ge, W. Zhou, A. Du, Z. Zhu, Porous Polyethersulfone-Supported Zeolitic Imidazolate Framework Membranes for Hydrogen Separation, The Journal of Physical Chemistry C, 116 (2012) 13264-13270.

[16] D. Nagaraju, D.G. Bhagat, R. Banerjee, U.K. Kharul, In situ growth of metal-organic frameworks on a porous ultrafiltration membrane for gas separation, Journal of Materials Chemistry A, 1 (2013). [17] F. Cacho-Bailo, B. Seoane, C. Téllez, J. Coronas, ZIF-8 continuous membrane on porous polysulfone for hydrogen separation, Journal of Membrane Science, 464 (2014) 119-126.

[18] I.F.J. Vankelecom, S.V.d. broeck, E. Merckx, Hilde Geerts, P. Grobet, J.B. Uytterhoeven, Silylation To Improve Incorporation of Zeolites in Polyimide Films, Journal of Physical Chemistry, 100 (1996) 3753-3758.

[19] X.Y. Chen, V.-T. Hoang, D. Rodrigue, S. Kaliaguinea, Optimization of continuous phase in aminofunctionalized metal-organic framework (MIL-53) based co-polyimide mixed matrix membranes for CO2/CH4 separation, RSC Advances, 3 (2013) 24266-24279.

[20] H. Siddique, E.Rundquist, Y.Bhole, L.G.Peeva, A.G.Livingston, Mixed matrix membranes for organic solvent nanofiltration, Journal of Membrane Science, 452 (2014) 354-366.

[21] C.J. Galvin, J. Genzer, Applications of surface-grafted macromolecules derived from postpolymerization modification reactions, Progress in Polymer Science, 37 (2012) 871-906.

[22] S. Tempelaar, L. Mespouille, O. Coulembier, P. Dubois, A.P. Dove, Synthesis and postpolymerisation modifications of aliphatic poly(carbonate)s prepared by ring-opening polymerisation, Chemical Society Reviews, 42 (2013) 1312--1336.

[23] G. Szekely, I.B. Valtcheva, J.F. Kim, A.G. Livingston, Molecularly imprinted organic solvent nanofiltration membranes - Revealing molecular recognition and solute rejection behaviour, Reactive and Functional Polymers, 86 (2015) 215-224.

[24] K.A. Günay, P. Theato, H.-A. Klok, Standing on the shoulders of Hermann Staudinger: Postpolymerization modification from past to present, ournal of Polymer Science Part A: Polymer Chemistry, 51 (2012) 1-28.

[25] Y.H. See-Toh, F.W. Limb, A.G. Livingston, Polymeric membranes for nanofiltration in polar aprotic solvents, Journal of Membrane Science, (2007) 3-10.

[26] K.C. Khulbe, C.Y. Feng, T. Matsuura, Pore Size, Pore Size Distribution, and Roughness at the Membrane Surface, in: Synthetic Polymeric Membranes, Springer Laboratory, 2008, pp. 101-139.

[27] A.R. Hassan, M.S.A. Munaim, Fabrication and characterization of integrally skinned-oriented highly selective charged asymmetric low pressure poly(ether sulfone) membranes for nanofiltration, Journal of Chemical Technology and Biotechnology, 87 (2011) 559-569. 
[28] J. Stawikowska, J.F. Kim, A.G. Livingston, Pore-flow calculations based on pore size distributions in polyimide membranes determined by a nanoprobe imaging technique, Chemical Engineering Science, 97 (2013) 81-95.

[29] D. Chaudhary, M.R. Went, K. Nakagawa, S.J. Buckman, J.P. Sullivan, Molecular pore size characterization within chitosan biopolymer using positron annihilation lifetime spectroscopy, Materials Letters, 64 (2010) 2635-2637.

[30] Y. Itoh, A. Shimazu, Y. Sadzuka, T. Sonobe, S. Itai, Novel method for stratum corneum pore size determination using positron annihilation lifetime spectroscopy, International Journal of Pharmaceutics, 358 (2008) 91-95.

[31] J. Stawikowska, A.G. Livingston, Nanoprobe imaging molecular scale pores in polymeric membranes, Journal of Membrane Science, 413-414 (2012) 1-16.

[32] Y.H. See-Toh, M. Silva, A. Livingston, Controlling molecular weight cut-off curves for highly solvent stable organic solvent nanofiltration (OSN) membranes, Journal of Membrane Science, 324 (2008) 220-232.

[33] I.B. Valtcheva, S.C. Kumbharkar, J.F. Kim, Y. Bhole, A.G. Livingston, Beyond polyimide: Crosslinked polybenzimidazole membranes for organic solvent nanofiltration (OSN) in harsh environments, Journal of Membrane Science, 457 (2014) 62-72.

[34] M.F.J. Solomon, Y. Bhole, A.G. Livingston, High flux membranes for organic solvent nanofiltration(OSN) - Interfacial polymerization with solvent activation, Journal of Membrane Science, 423-424 (2012) 371-382.

[35] W.R. Bowen, J.S. Welfoot, Modelling of membrane nanofiltration-pore size distribution effects, Chemical Engineering Science, 57 (2002) 1393-1407.

[36] G. Belfort, J.M. Pimbley, A. Greiner, K.Y. Chung, Diagnosis of membrane fouling using a rotating annular filter. 1. Cell culture media, Journal of Membrane Science, 77 (1993) 1-22.

[37] A.L. Zydney, P. Aimar, M. Meireles, J.M. Pimbley, G. Belfort, Use of the log-normal probability density function to analyze membrane pore size distributions: functional forms and discrepancies, Journal of Membrane Science, 91 (1994) 293-298.

[38] W.R. Bowen, J.S. Welfoot, Modelling the performance of membrane nanofiltration-critical assessment and model development, Chemical Engineering Science, 57 (2002) 1121-1137.

[39] W.R. Bowen, J.S. Welfoot, Predictive modelling of nanofiltration: membrane specification and process optimisation, Desalination, 147 (2002) 197-203.

[40] P. Silva, A.G. Livingston, Effect of solute concentration and mass transfer limitations on transport in organic solvent nanofiltration - partially rejected solute, Journal of Membrane Science, 280 (2006) 889-898.

[41] J.L.C. Santos, P. de Beukelaar, I.F.J. Vankelecom, S. Velizarov, J.G. Crespo, Effect of solute geometry and orientation on the rejection of uncharged compounds by nanofiltration, Separation and Purification Technology, 50 (2006) 122-131.

[42] J.A. Otero, O. Mazarrasa, J. Villasante, V. Silva, P. Prádanos, J.I. Calvo, A. Hernández, Three independent ways to obtain information on pore size distributions of nanofiltration membranes, Journal of Membrane Science, 309 (2008) 17-27.

[43] W.M. Deen, Hindered transport of large molecules in liquid-filled pores, AIChE Journal, 33 (1987) 1409-1425.

[44] W.R. Bowen, A.O. Sharif, Transport through Microfiltration Membranes-Particle Hydrodynamics and Flux Reduction, Journal of Colloid and Interface Science, 168 (1994) 414-421.

[45] W.R. Bowen, A.W. Mohammad, N. Hilal, Characterisation of nanofiltration membranes for predictive purposes - use of salts, uncharged solutes and atomic force microscopy, Journal of Membrane Science, 126 (1997) 91-105.

[46] C.R. Wilke, P. Chang, Correlation of diffusion coefficients in dilute solutions, AIChE Journal, 1 (1955) 264-270. 
[47] Y.H. Zhao, M.H. Abraham, A.M. Zissimos, Fast Calculation of van der Waals Volume as a Sum of Atomic and Bond Contributions and Its Application to Drug Compounds, The Journal of Organic Chemistry, 68 (2003) 7368-7373.

[48] N. Janssens, L.H. Wee, S. Bajpe, E. Breynaert, C.E.A. Kirschhock, J.A. Martens, Recovery and reuse of heteropolyacid catalyst in liquid reaction medium through reversible encapsulation in $\mathrm{CU}_{3}(\mathrm{BTC})_{2}$ metal-organic framework, Chemical Science, 3 (2012) 1847-1850.

[49] W.R. Bowen, J.S. Welfoot, Modelling the performance of membrane nanofiltration-critical assessment and model development, Chemical Engineering Science, 57 (2002) 1121- 1137.

[50] J. Campbell, R.P. Davies, D.C. Braddock, A.G. Livingston, Improving the Permeance of Hybrid Polymer/ Metal Organic Framework (MOF) Membranes for Organic Solvent Nanofiltration (OSN) Development of MOF Thin Films via Interfacial Synthesis, Journal of Material Chemistry A, 3 (2015) 9668-9674.

[51] Y.H.S. Toh, X.X. Loh, K. Li, A. Bismarck, A.G. Livingston, In search of a standard method for the characterisation of organic solvent nanofiltration membranes, Journal of Membrane Science, 291 (2007) 120-125. 